\title{
Small Cell Lung Cancer with Extensive Cutaneous and Gastric Metastases
}

\author{
Juichiro Maeda, Mitsutomi Mryake, Kazuhiko Tokita, Noriaki Iwahashi, Takashi Nakano, \\ Shinsuke Tamura, Toshikazu Hada and Kazuya Higashino
}

\begin{abstract}
A case of a 60-year-old Japanese woman with small cell lung cancer involving skin and stomach is reported. She was diagnosed as primary small cell lung cancer accompanied by extensive cutaneous metastases. Three months after the last chemotherapy, she complained of nausea and vomiting. Brain CT scan showed no evidence of central nervous system involvement. Upper gastrointestinal study and upper gastrointestinal fiberscopy revealed multiple metastatic gastric tumors. Skin and stomach are uncommon metastatic sites for any malignancy. Furthermore, only a few cases with gastric metastasis could be diagnosed during their lifetime.

(Internal Medicine 31: 1325-1328, 1992)
\end{abstract}

Key words: submucosal gastric tumor, bull's eye lesion, target lesion

\section{Introduction}

Primary small cell lung cancer (SCLC), which comprises about one-fourth of all lung cancer, frequently metastasizes to lung, brain, liver and adrenal gland. It is uncommon for lung cancer to metastasize to skin or stomach. Moreover, it is extremely rare that gastric metastasis is manifested in life $(1,2)$; and most metastatic gastric tumors have been found at autopsy (3). We report here a case of SCLC with extensive cutaneous and gastric metastases that could be diagnosed during the patient's lifetime. When patients of any type of malignancy complain of upper digestive tract symptoms, gastric metastasis, as well as brain metastasis and side effects of chemotherapy, must be considered as possible causes of these symptoms.

\section{Case Report}

A 60-year-old Japanese woman was admitted to the dermatology ward of our hospital because of multiple skin tumors. She was well until three months earlier when she noticed several skin tumors on her face and anterior chest wall. They had gradually increased in number and spread to involve the scalp, neck, back, abdominal wall and upper and lower extremities. Skin tumors were about 80 in number and varied from 0.5 to $2.0 \mathrm{~cm}$ in diameter. Each tumor was nodular, movable, painless and covered by intact epidermis. Ulceration was not presented. Some of the overlying skin was reddish in color (Fig. 1). The histological examination of the specimen obtained from biopsy of a skin tumor showed metastatic small cell carcinoma (Fig. 2). She was transferred to our ward for further evaluation of the primary site and for systemic chemotherapy.

She was well nourished and had no respiratory or upper gastrointestinal symptoms. Physical examination on admission showed no significant findings except for the extensive skin tumors. Her peripheral blood cell count was normal. No abnormalities were found in the liver and renal function tests. Tumor markers, such as AFP, CEA and NSE were all normal. Arterial blood gas analysis at room air showed moderate hypoxemia (pH 7.38, $\mathrm{PacO}_{2}$ 38.6 Torr, $\mathrm{PaO}_{2}$ 64.9 Torr). A test for occult blood in the stool was negative.

An X-ray film of the chest taken on admission to the dermatology ward revealed tumors in the right lower and left upper lung fields (Fig. 3, left). Chest CT revealed a main tumor at right $\mathrm{S}^{7}$ (Fig. 3, right). Bronchoscopy was performed. The specimens obtained from both tumors by transbronchial lung biopsy (TBLB) were histologically diagnosed as small cell carcinoma (Fig. 4). Other examinations for primary and metastatic sites, such as upper gastrointestinal study (UGI study), barium enema, abdominal ultrasonography, abdominal and pelvic $\mathrm{CT}$ scan revealed no significant findings.

From the Third Department of Internal Medicine, Hyogo College of Medicine, Hyogo Received for publication March 2, 1992; Accepted for publication September 25, 1992

Reprint requests should be addressed to Dr. Juichiro Maeda, the Third Department of Internal Medicine, Hyogo College of Medicine, 1-1, Mukogawa-cho, Nishinomiya, Hyogo 663, Japan 
There were also no remarkable findings in the gynecological and otopharyngeal examinations. She was clinically diagnosed as SCLC accompanied by multiple cutaneous metastases. In spite of intensive chemotherapy, consisting of cisplatinum, etoposide and doxorubicin,

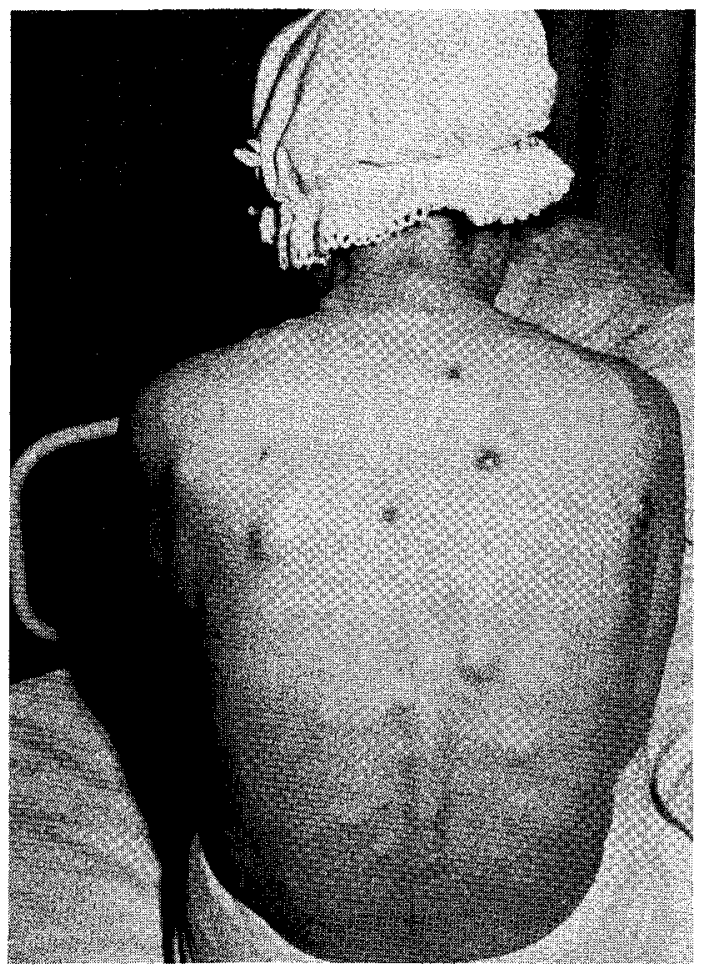

Fig. 1. Metastatic cutaneous tumors on her back. Multiple subcutaneous tumors covered by normal epidermis spread on her back. The overlying skin is reddish in color, in some. the lung and skin tumors increased, not in size, but in number. Although another chemtherapy regimen consisting of cyclophosphamide, doxorubicin and vincristine was prescribed, it was not effective. A plain chest film showed worsening of the primary tumor and the development of small nodules throughout both lungs. Three months after the last chemotherapy, she complained of nausea and vomiting. She had no history of tarry stool and a test for occult blood in the stool was negative. Although a brain CT was performed for these symptoms, no evidence of central nervous system involvement was found. Judging from the interval after the last chemotherapy, the side effects of anticancer drugs were unlikely the cause of these symptoms. Upper gastrointestinal study revealed multiple barium

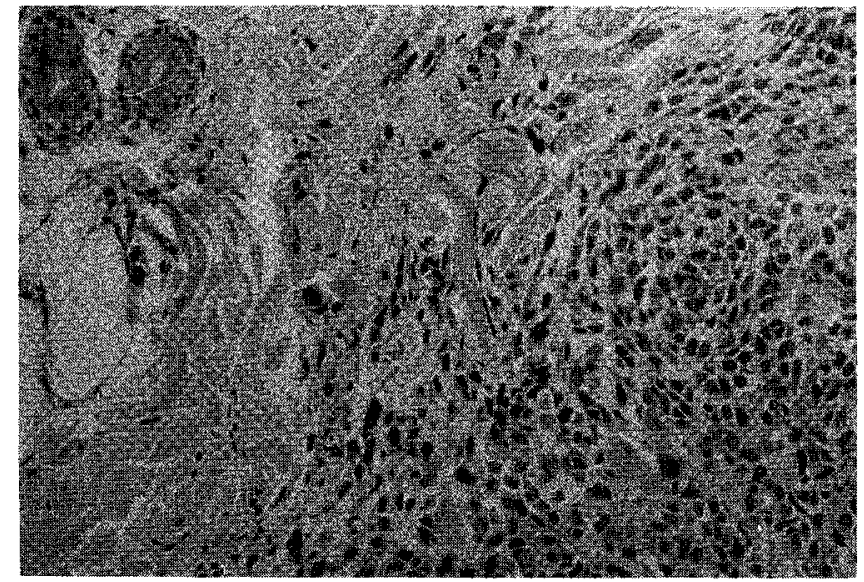

Fig. 2. The histological finding of the specimen obtained by skin tumor biopsy shows metastatic small cell carcinoma. Tumor cells are situated in the subcutaneous layer (HE stain, $\times 100$ ).

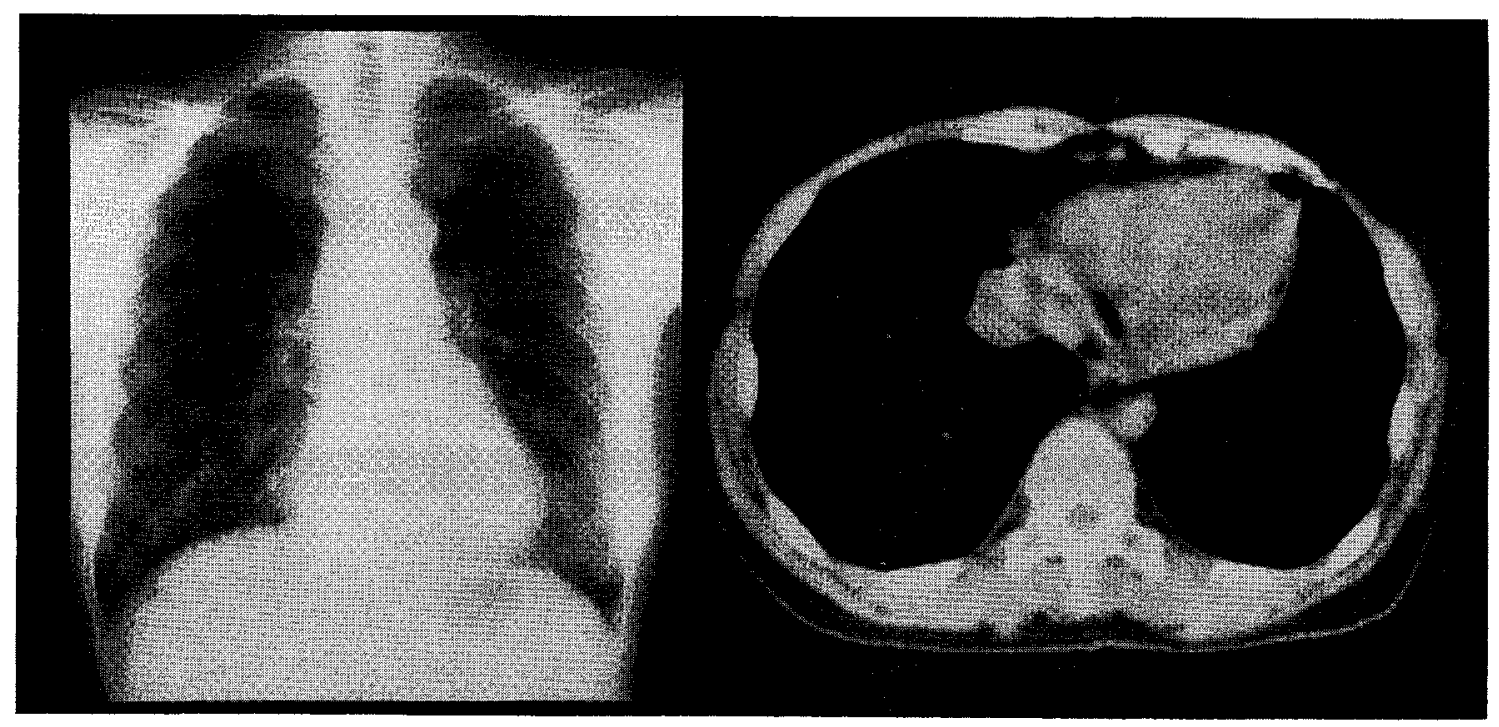

Fig. 3. Left; A plain chest film taken on admission to the dermatology ward reveals tumor shadows in the right lower and the left upper lung fields. Right; A chest CT scan shows a main tumor in the right lower lung field on the plain chest film located at right $\mathrm{S}^{7}$. 


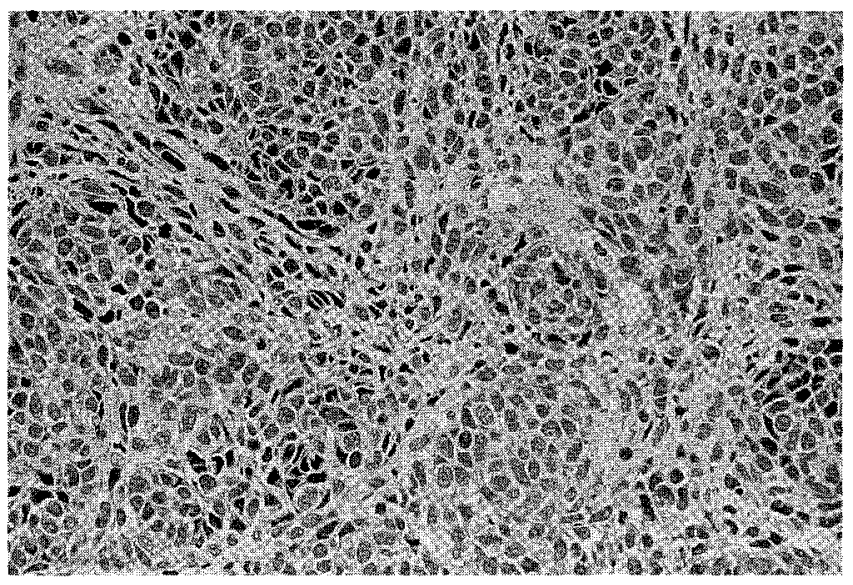

Fig. 4. The histological finding of the specimen from the tumor at right $\mathrm{S}^{7}$ shows small cell carcinoma. The specimen was obtained by TBLB (HE stain, $\times 100$ ).

defects throughout the whole stomach. These were radiologically described as "bull's eye" or "target" lesions (Fig. 5, left). Gastrointestinal fiberscopy (GIF) showed multiple submucosal tumors (Fig. 5, right). Histological examination of these tumors showed metastatic small cell carcinoma (Fig. 6). Systemic chemotherapy was not performed because of her poor condition. Her respiratory condition gradually worsened, and she died of respiratory failure. Autopsy was not permitted.

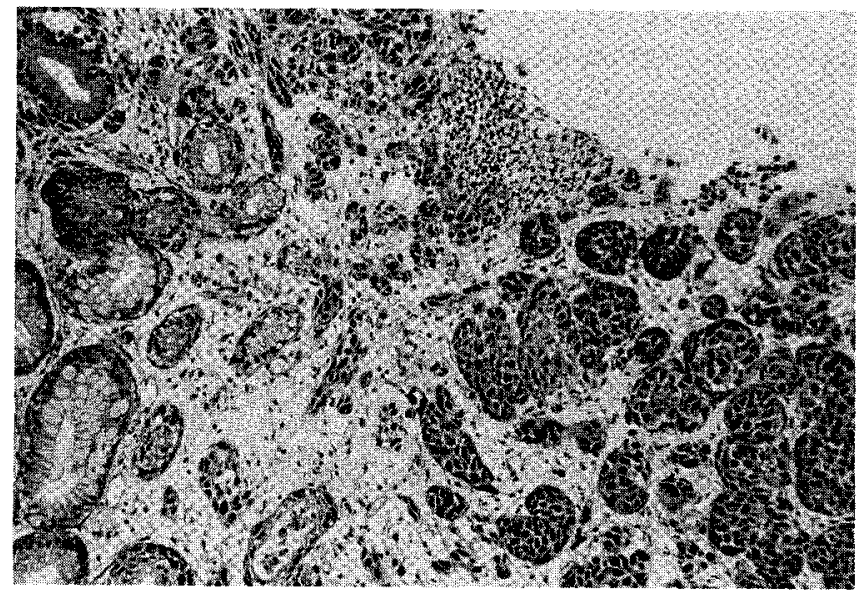

Fig. 6. A microscopical finding of the specimen obtained by the biopsy of a submucosal tumor under GIF shows metastatic small cell carcinoma. Tumor cells are mainly situated in the submucosal layer (HE stain, $\times 100$ )

\section{Discussion}

SCLC has a tendency to progress rapidly and to metastasize early and extensively. Common metastatic sites include lung, liver, bone, adrenal gland and central nervous system. In any malignancy, gastric metastasis has rarely been reported, and most of these were proven at autopsy. Some authors have reported them with a frequency of $2 \%$ of all autopsies $(3,4)$. Gastric metastasis from lung cancer in autopsy cases has been demonstrated with a frequency of 2 to $9 \%(3,4)$. Moreover, it is

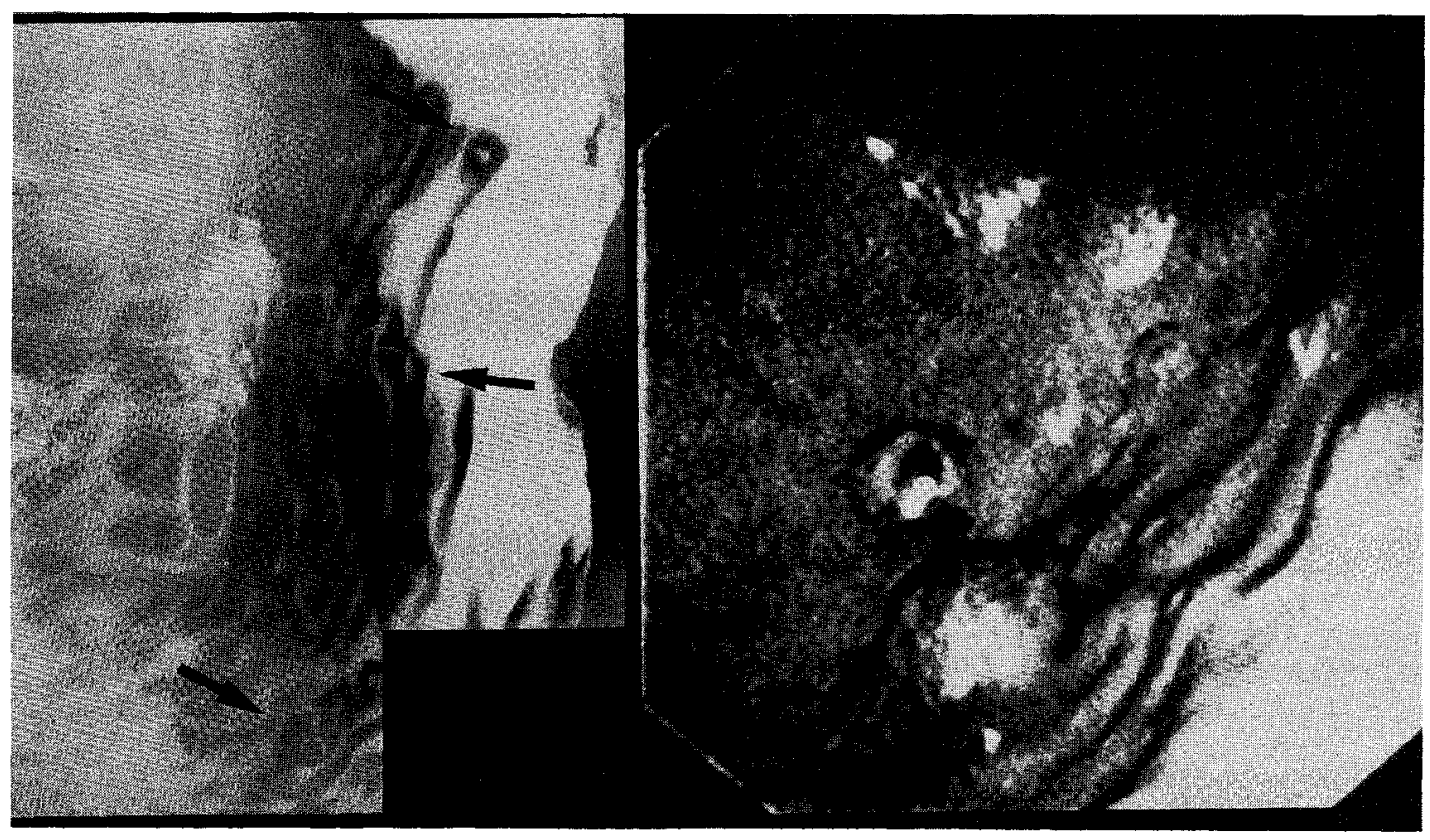

Fig. 5. Left; UGI study shows several "bull's eye" or "target" lesions in the body of stomach (shown by arrows). Right; A finding of GIF. Multiple submucosal tumors are shown on the major curvature in the body of the stomach. 


\section{Maeda et al}

extremely rare that gastric metastasis is manifested during the patient's lifetime $(1,3,4)$.

Some mechanisms of gastric metastasis are considered as follows: 1) direct invasion, such as in pancreatic cancer or esophageal cancer, 2) disseminated involvement of the peritoneum, such as in ovarian cancer, 3) hematogeneous metastasis most often occurring in lung cancer $(2,5,6)$. There have been no reports to clarify the reason for the rarity of gastric metastasis from lung cancer. Although the stomach receives plenty of blood supply, the mechanism of gastric metastasis can not be explained only by this anatomical factor. Recently, it is suspected that some types of cytokines may affect the organ specificity in hematogeneous metastasis $(7,8)$. Hematogeneous metastatic tumor cells to the stomach are situated in the submucosal layer and develop into submucosal tumors. Radiologically, these metastatic gastric tumors are described as "bull's eye" or "target" lesions, which are the characteristic findings on UGI study $(4,9)$. Endoscopically, these lesions are often described as submucosal tumors with bridging folds and small ulcerations on the top $(3,5,6)$. Patients with gastric metastases from any types of malignancy are often asymptomatic unless the metastases invade the gastric mucosa or serosa, or occupy the gastric lumen $(1,3,9,10)$. Moreover, further evaluation of gastrointestinal tract is unlikely to be performed because the symptoms, such as nausea and vomiting, are usually regarded as side effects of chemotherapy or as symptoms of the involvement of the central nervous system. This fact makes gastric metastasis difficult to confirm during life and also contributes to the poor prognosis of cases with gastric metastases. Clinicians must consider the possibility of gastric metastasis as one of the causes of digestive tract symptoms during the treatment of any malignancy.

Skin is also an uncommon metastatic site for any malignancy. The reported incidence is 2 to $5 \%$ of all patients with internal malignancies $(11,12)$. In men, lung cancer is the most common primary site of cutaneous metastasis, and it is breast cancer in women (13). Although the anterior chest wall and upper extremities are the most common locations for occurrence of lung cancer skin metastases, extensive cutaneous metastases, as in our case, are very rare $(13,15)$. Metastatic cutaneous tumors are often characterized as nodular, painless and mobile tumors usually covered by intact epidermis
$(11,16)$. Ulceration is uncommon. Cutaneous metastasis is believed to indicate a poor prognosis because most occur in the advanced stages of the primary malignancies.

In summary, a case of SCLC with extensive cutaneous and multiple gastric metastases is reported. This is one of the rare case reports of gastric metastasis manifested during a patient's lifetime. We emphasize that gastrointestinal tract examination should be performed for upper gastrointestinal tract symptoms during treatment of any malignancy $(15,16)$.

\section{References}

1) Geboes K, De Jaeger E, Rutgeers P, Vantrappen G. Symptomatic gastrointestinal metastases from malignant melanoma. J Clin Gastroenterol 10: 64, 1988.

2) Radin DR, Halls JM. Cavitating metastases of the stomach and duodenum. Comput Tomogr 11: 283, 1987.

3) Schmidt G, Borsch G, von Licbe S, Bohm E. Gastric perforation secondary to metastatic bronchogenic carcinoma. Hepatogastroenterology 32: 103, 1985.

4) Rubin SA, Davis M. "Bull's eye" or "target" lesions of the stomach secondary to carcinoma of the lung. Am J Gastroenterol 80: 67,1985

5) Lynch P, Green L, Jordan PH, Graham DY. Hepatocellular carcinoma metastatic to the stomach presenting as bleeding multiple craterogenic ulcers. Am J Gastroenterol 84: 653, 1989.

6) Higgins PM, B.M., B.Ch. Pyloric obstruction due to a metastatic deposit from carcinoma of the bronchus. Can J Surg 5: 438, 1962.

7) Postlewaite AE, Lachman LB, Mainardi CL, et al. Interleukin 1 stimulation of collagenase production by cultured fibroblasts. J Exp Med 157: 801, 1983.

8) Thomas KA, Rios-Candeiore M, Gimenez-Gallego G, et al. Pure brain-dcrived acidic fibroblast growth factor is a potent angiogenic vascular endothelial cell mitogen with sequence homology to interleukin 1. Proc Natl Acad Sci USA 82: 6409, 1985.

9) Blessing K, Park K, McLaren K, Eremin O. Gastrointestinal involvement with metastatic melanoma: Clinical and pathological features. R Coll Surg Edinb 31: 293, 1986.

10) Cascinu S, Fedeli SL, Fedeli A, Catalano G. Gastric metastases from breast cancer: A case report. Tumori 75: 150, 1989.

11) White JW Jr. Evaluating cancer metastatic to the skin. Geriatrics 40: 67, 1985.

12) Fox JL, Berman B, Prioleau PG. Skin metastases from small cell carcinoma of the lung. J Dermatol Oncol 9: 451, 1983.

13) McKee PH. Cutaneous metastases. Cutan Pathol 12: 239, 1985.

14) Spencer PS, Helm TN. Skin metastases in cancer patients. Cutis 20: $119,1987$.

15) Tharakaram S. Metastases to the skin. Int J Dermatol 27: 240, 1988.

16) Dreizen $S$, Dhingra HM, Chiuten DF, Umsawasdi $T$, Valdivicso M. Cutaneous and subcutaneous metastases of lung cancer; Clinical characteristics. Postgrad Med 80: 111, 1986. 